\title{
Venous Thromboembolism in Pediatric Cancer Patients with Central Venous Catheter-A Systematic Review and Meta-analysis
}

\author{
Rasmus Søgaard Hansen, MD ${ }^{1}$ Mads Nybo, MD, $\mathrm{PhD}^{1}$ Anne-Mette Hvas, MD, $\mathrm{PhD}^{2,3}$ \\ ${ }^{1}$ Department of Clinical Biochemistry and Pharmacology, Odense \\ University Hospital, Odense, Denmark \\ 2 Department of Clinical Biochemistry, Aarhus University Hospital, \\ Aarhus, Denmark \\ ${ }^{3}$ Department of Clinical Medicine, Aarhus University, Aarhus, \\ Address for correspondence Rasmus Søgaard Hansen, MD, \\ Department of Clinical Biochemistry and Pharmacology, Odense \\ University Hospital, J.B. Winsløws Vej 4, DK-5000 Odense C, Denmark \\ (e-mail: Rasmus.Sogaard.Hansen@rsyd.dk).
} Denmark

Semin Thromb Hemost 2021;47:920-930.

\begin{abstract}
Keywords

- central venous catheter

- pediatric

- thromboembolism

- neoplasm

Pediatric cancer patients hold an increased risk of venous thromboembolism (VTE) due to their cancer. Central venous catheters (CVCs) further increase the VTE risk. This systematic literature review elucidates the VTE incidence in pediatric cancer patients with CVC. MEDLINE and EMBASE were searched in August 2020 without time limits. We included studies reporting original data on patients $\leq 18$ years with any CVC type and any cancer type, who were examined for VTE with $\geq 7$ days follow-up. In total, 682 unique records were identified, whereof 189 studies were assessed in full text. Altogether, 25 studies were included, containing 2,318 pediatric cancer patients with CVC, of which $17 \%$ suffered VTE. Fifteen studies $(n=1,551)$ described CVCrelated VTE and reported 11\% CVC-related VTE. Concerning cancer type, 991 children suffered from acute lymphoblastic leukemia (ALL) and 616 from solid tumors. Metaanalysis revealed VTE incidence (95\% confidence interval) of $21 \%$ (8-37) for ALL and $7 \%$ $(0.1-17)$ for solid tumors. Additionally, $20 \%$ of children with tunneled or nontunneled CVC and $12 \%$ of children with implantable ports suffered VTE. In conclusion, pediatric cancer patients with CVC have substantial VTE risk. Children with ALL and CVC have higher VTE incidence than children with solid tumors and CVC. Implantable port catheter should be preferred over tunneled or nontunneled CVC to reduce VTE risk. Thrombophilia investigation does not seem relevant in pediatric cancer patients with CVC and VTE. To prevent VTE, intensified catheter care is recommended, especially in children with ALL.
\end{abstract}

The incidence of pediatric cancer is 156 per million personyears worldwide, with leukemia being the most prevalent. ${ }^{1} \mathrm{~A}$ relationship between cancer and venous thromboembolism (VTE) has been known since 1865 . $^{2}$ Development of VTE is associated with lower survival, especially for children with hematological cancers. ${ }^{3}$ The VTE prevalence is around $8 \%$ in

published online September 2, 2021
Issue Theme Cardiovascular and Thromboembolic Diseases in Oncology: Novel Aspects and Revisited Issues; Antonella Tufano, MD, PhD, Antonio Coppola, MD, Maurizio Galderisi, MD, and Massimo Franchini, MD pediatric cancer patients. ${ }^{4}$ Numerous risk factors for VTE in pediatric cancer patients have been identified, including cancer type, ${ }^{5}$ chemotherapy, ${ }^{6}$ and central venous catheters (CVCs). ${ }^{7,8}$ CVCs are frequently used in pediatric cancer patients to administer chemotherapy, transfusions, and parenteral nutrition. Although helpful, CVCs also pose a risk for (c) 2021. Thieme. All rights reserved. Thieme Medical Publishers, Inc., 333 Seventh Avenue, 18th Floor, New York, NY 10001, USA
DOI https://doi.org/ 10.1055/s-0041-1729886. ISSN 0094-6176. 
VTE development. ${ }^{8}$ In adult cancer patients, the incidence of catheter-associated VTE is 6 to $15 \% .^{9,10}$ Although CVC is a confirmed VTE risk factor in pediatric cancer patients, ${ }^{8}$ thorough research on this is lacking, especially regarding cancer and catheter types at risk along with strategies for thromboprophylaxis.

Thus, the aim of the present systematic review was to investigate the incidence of VTE in pediatric cancer patients with CVC inserted. Additionally, the employed catheter care regimens, VTE prophylaxis regimens used, and the role of thrombophilia are elucidated.

\section{Materials and Methods}

This review was conducted according to a prespecified protocol (Supplementary Material 1, available in the online version only) and the Preferred Reporting Items for Systematic reviews and Meta-Analyses (PRISMA) statement. ${ }^{11}$ A systematic search of the databases MEDLINE (PubMed) and Ovid (EMBASE) was performed November $12^{\text {th }}, 2019$ with no restrictions on publication period but limited to original studies on humans published in English. The search was updated on August $4^{\text {th }}, 2020$. The search strategy included MeSH terms related to any type of cancer, CVC, and VTE, but restricted on age (child: birth-18 years).

The inclusion criteria were prospective studies reporting original data, English language, and studies on pediatric patients ( $\leq 18$ years) with any type of CVC and any type of cancer, which reported investigation for VTE. The exclusion criteria were reviews, guidelines, letters without original data, editorials, studies with less than 10 cases, posters or conference abstracts, ongoing trials, in vitro studies, and animal studies. Moreover, studies with less than 7 days follow-up were excluded to ensure sufficient observation time for VTE development.

To validate the inclusion and exclusion criteria, a random sample of 50 abstracts was screened and discussed by A.M.H. and R.S.H. until consensus was achieved. Remaining records identified were screened by title and abstract by RSH. To validate the inclusion and exclusion criteria for studies in full text, 12 full-text studies were evaluated and discussed by A. M.H. and R.S.H. until consensus was achieved. The remaining full-text studies were evaluated by R.S.H. Data extraction was performed by R.S.H. and all extracted data were validated by A.M.H. and M.N.

\section{Cancer Diagnosis}

Cancer diagnosis was divided into the following five classifications: acute lymphoblastic leukemia (ALL), non-ALL hematological cancer, solid tumors, and any cancer if the diagnosis was not specified and any hematological cancer if the number of ALL cases was not specified.

\section{Catheter Types}

Catheters were divided into three classifications: tunneled or nontunneled CVC, implantable ports, and peripherally inserted central catheters (PICCs).

\section{Venous Thromboembolism}

VTE was defined as a clinical episode of VTE at any site, including superficial and deep vein thrombosis and pulmonary embolism. If the study reported a VTE, but no definition of VTE was provided, the incidence was also included in this review. Only studies clearly specifying VTE episodes as CVC related, were counted as CVC-related VTE. The approaches for VTE diagnosis are described for all included studies in -Table 1.

\section{Study Quality Assessment}

For all included studies, the Study Quality Assessment Tools checklist provided by National Heart, Lung and Blood Institute was completed by R.S.H., M.N., and A.M. ${ }^{12}$ The checklist for observational cohort and cross-sectional studies, case-control studies, and systematic reviews and meta-analysis were used, respectively. The study quality was assessed with regard to the purpose of this systematic review. Each study was rated good, fair, or poor according to the estimated risk of bias. After individual assessment, R.S.H., M.N., and A.M. discussed any rating disagreement until consensus was achieved.

\section{Statistical Analyses}

For VTE incidence calculation, a random-effects model was chosen since the included studies were heterogeneous and a high level of variance therefore had to be taken into consideration. $^{13}$

The extracted incidence proportions were transformed by the double arcsine model to calculate an overall mean incidence with $95 \%$ confidence intervals (CIs) and presented in a forest plot. ${ }^{13}$ Statistical heterogeneity was assessed by $I^{2}$ statistics. ${ }^{14,15}$

Publication bias was assessed by funnel plots. ${ }^{16}$ Statistical analyses and meta-analysis were performed using MetaXL (EpiGear International, Sunrise Beach, Queensland, Australia).

\section{Results}

The inclusion and exclusion process is depicted in - Fig. 1. In total, 682 unique records were identified. After screening all abstracts and titles, 189 studies were assessed in full text for eligibility. Among these, 25 studies were included in the qualitative synthesis, while 17 studies reporting VTE for patients with ALL or solid tumors were also included in a quantitative synthesis (meta-analysis). Of the 25 included studies, three were randomized controlled trials, ${ }^{17-19}$ one was a case-control study, ${ }^{20}$ and the remaining 21 studies were cohort studies. ${ }^{21-41}$ of the 25 included studies, six were rated good, ${ }^{17,23,30,31,38,40}$ seven fair $^{18,22,25,26,28,36,37}$, and 12 poor quality. ${ }^{19-21,24,27,29,32-35,39,41}$

In total, 2,318 pediatric cancer patients with a catheter inserted were included in the studies, of which 991 were classified as ALL, 616 solid tumors, 352 any cancer, 211 any hematological cancer, and 148 as non-ALL hematological cancer.

The total number of catheters could not be calculated due to lack of data in the included studies. In total, results on 629 tunneled or nontunneled CVCs, 444 implantable ports, 28 PICCs, and 86 nonspecified catheters were reported. Study design, characteristics of patients along with exposure and outcome are presented in -Table $\mathbf{1}$. 
922 VTE in Pediatric Cancer Patients with CVC Hansen et al.

Table 1 Summary of included studies investigating venous thromboembolism in pediatric cancer patients with a catheter inserted $(n=25)$

\begin{tabular}{|c|c|c|c|c|}
\hline $\begin{array}{l}\text { Study } \\
\text { Author } \\
\text { Year } \\
\text { Quality rating }\end{array}$ & $\begin{array}{l}\text { Design } \\
\text { Follow-up } \\
\text { time (FT) }\end{array}$ & $\begin{array}{l}\text { Characteristics } \\
\text { Patients, } n \\
\text { Age }\end{array}$ & $\begin{array}{l}\text { Exposure } \\
\text { Catheter type, } n \\
\text { Catheter duration } \\
\text { Catheter care }\end{array}$ & $\begin{array}{l}\text { Outcome } \\
\text { Venous thromboembolism, } n \\
\text { Diagnostic approach }\end{array}$ \\
\hline $\begin{array}{l}\text { Previtali et al } 2019 \\
\text { Good }^{40}\end{array}$ & $\begin{array}{l}\text { Cohort, } \\
\text { single center } \\
\text { FT: } \leq 164 \text { days }\end{array}$ & $\begin{array}{l}\text { Solid tumors, } n=63 \\
\text { Any cancer, } n=29 \\
\text { Hema cancer, } n=11 \\
\text { Age (median): } 10.5 \text { years }\end{array}$ & $\begin{array}{l}\text { Type: Port, } n=74, \text { PICC, } n=28 \text {, } \\
\text { CVC, } n=11 \\
\text { Duration (median): } 87 \text { days } \\
\text { Care: NR } \\
\text { VTE-therapy: Anticoagulant therapy } \\
\text { (not specified). }\end{array}$ & $\begin{array}{l}\text { Symptomatic, } n=0 \\
\text { Asymptomatic, } n=3 \\
\text { (non-ALL hema cancer, } n=1 \text {, } \\
\text { solid tumors, } n=2 \text { ) } \\
\text { Port related, } n=2 \\
\text { PICC related, } n=1 \\
\text { Catheter removal: NR } \\
\text { Diagnosis: ultrasound (US) at } 15 \text {, } \\
\text { 30, and } 90 \text { days after implantation. } \\
\text { In total, } 91 \text { of } 113 \text { catheters } \\
\text { completed all } 90 \text { days. }\end{array}$ \\
\hline $\begin{array}{l}\text { Östlund et al } 2019 \\
\text { Fair }^{36}\end{array}$ & $\begin{array}{l}\text { Cohort, } \\
\text { single center } \\
\text { FT: NR }\end{array}$ & $\begin{array}{l}\text { Any cancer, } n=N R \\
\text { Age: NR }\end{array}$ & $\begin{array}{l}\text { Type: CVC, } n=19 \\
\text { Duration: NR } \\
\text { Care: NR. }\end{array}$ & $\begin{array}{l}\text { Thrombosis, } n=3 \\
\text { Symptomatic: NR } \\
\text { Asymptomatic: NR } \\
\text { Catheter related: NR } \\
\text { Catheter removal: NR } \\
\text { Diagnosis: Followed for clinical signs } \\
\text { and symptoms. Confirmed by US. } \\
\text { At catheter removal US was } \\
\text { performed. }\end{array}$ \\
\hline $\begin{array}{l}\text { Schoot et al } 2016 \\
\text { Good }^{17}\end{array}$ & $\begin{array}{l}\mathrm{RCT} \\
\text { double blind } \\
\mathrm{FT}: \leq 6 \text { months }\end{array}$ & $\begin{array}{l}\text { Solid tumors, } n=157 \\
\text { Hema cancer, } n=148 \\
\text { Age (median): } 9 \text { years }\end{array}$ & $\begin{array}{l}\text { Type: CVC, } n=18 \text {, Port, } n=287 \\
\text { Duration: } \\
168 \text { patients: } 6 \text { months } \\
137 \text { patients: } 56 \text { days (median) } \\
\text { Care: Once every } 1-6 \text { week } \\
1.5-3.0 \mathrm{~mL} \text { lock solution (either } \\
70 \% \text { ethanol or } 100 \mathrm{IU} / \mathrm{mL} \text { heparin). }\end{array}$ & $\begin{array}{l}\text { Symptomatic, } n=8 \\
\text { (hema cancer, } n=7 \text {, solid tumors, } \\
n=1 \text { ). } \\
\text { Asymptomatic/total, } n=12 / 185 \\
\text { (hema cancer, } n=7 \text {, solid tumors, } \\
n=5 \text { ) } \\
\text { Catheter related, } n=14 \\
\text { Catheter removal, } n=1 \\
\text { Diagnosis: Close monitoring. } \\
\text { If clinically suspected, confirmed by } \\
\text { imaging. In } 185 \text { patients, bilateral } \\
\text { US was performed at study } \\
\text { completion. }\end{array}$ \\
\hline $\begin{array}{l}\text { Kulkarni et al } 2015 \\
\text { Poor }^{21}\end{array}$ & $\begin{array}{l}\text { Cohort, } \\
\text { multicenter } \\
\text { FT: } \geq 6 \text { months }\end{array}$ & $\begin{array}{l}\text { ALL, } n=101 \\
\text { Any cancer, } n=67 \\
\text { Non-ALL hema cancer, } n=57 \\
\text { Solid tumors, } n=30 \\
\text { Age (median): } 15 \text { years }\end{array}$ & $\begin{array}{l}\text { Type: CVC, } n=\mathrm{NR} \text {, Port, } n=\mathrm{NR} \text {, } \\
\text { PICC, } n=\mathrm{NR} \\
\text { Duration: NR } \\
\text { Care: NR. }\end{array}$ & $\begin{array}{l}\text { Symptomatic, } n=75 \\
\text { (ALL, } n=29 \text {, any cancer, } n=23 \\
\text { non-ALL hema cancer, } n=20 \text {, } \\
\text { solid tumors, } n=3 \text { ) } \\
\text { Asymptomatic, } n=9 \\
\text { (ALL, } n=5 \text {, solid tumors, } n=2 \\
\text { any cancer, } n=1 \\
\text { non-ALL hema cancer, } n=1 \text { ) } \\
\text { Diagnosis: Symptomatic: If clinically } \\
\text { suspected, confirmed by imaging. } \\
\text { Asymptomatic: Incidentally } \\
\text { detected by imaging. }\end{array}$ \\
\hline $\begin{array}{l}\text { Abate et al } 2014 \\
\text { Fair }^{37}\end{array}$ & $\begin{array}{l}\text { Cohort, } \\
\text { single center } \\
\text { FT: NR }\end{array}$ & $\begin{array}{l}\text { Solid tumors, } n=155 \\
\text { Age (median): } 13.2 \text { years }\end{array}$ & $\begin{array}{l}\text { Type: CVC, } n=153 \text {, Port, } n=2 \\
\text { Duration (median): } 281 \text { days } \\
\text { Care: Flushing on alternate days or } \\
\text { at every use with } 20 \mathrm{~mL} \text { saline fol- } \\
\text { lowed by } 3 \mathrm{~mL} \mathrm{NaCl} 0.9 \% \text { with } \\
\text { heparin } 200 \mathrm{IU} / \mathrm{mL} \\
\text { VTE therapy: Following published } \\
\text { guidelines (not specified). } \\
\text { VTE prophylaxis: LMWH in } 85 \\
\text { patients (only patients }>10 \text { years } \\
\text { old). }\end{array}$ & $\begin{array}{l}\text { Symptomatic, } n=1 \\
\text { Asymptomatic: NR } \\
\text { Port related, } n=1 \\
\text { Catheter removal, } n=1 \\
\text { Diagnosis: If symptomatic, } \\
\text { confirmed by US, CT, or MRI. }\end{array}$ \\
\hline $\begin{array}{l}\text { Albisetti et al } 2013 \\
\text { Good }^{38}\end{array}$ & $\begin{array}{l}\text { Cohort, } \\
\text { single center } \\
\text { FT: } \leq 7.2 \text { years }\end{array}$ & $\begin{array}{l}\text { Solid tumors, } n=48 \\
\text { ALL, } n=38 \\
\text { non-ALL hema cancer, } n=16 \\
\text { Age: NR }\end{array}$ & $\begin{array}{l}\text { Type: Port, } n=\mathrm{NR} \\
\text { Duration: NR } \\
\text { Care: NR. }\end{array}$ & $\begin{array}{l}\text { Symptomatic, } n=5 \\
\text { (ALL, } n=3 \text {, non-ALL hema cancer, } \\
n=1 \text {, solid tumors, } n=1 \text { ) } \\
\text { Symptomatic, events, } n=7 \\
\text { (one ALL patient had VTE x3) } \\
\text { Asymptomatic, } n=31 \\
\text { (solid tumors, } n=20, \text { ALL, } n=11 \text { ) } \\
\text { Catheter related: } 36 \\
\text { Catheter removal: NR } \\
\text { Diagnosis: MRV. }\end{array}$ \\
\hline
\end{tabular}


Table 1 (Continued)

\begin{tabular}{|c|c|c|c|c|}
\hline $\begin{array}{l}\text { Study } \\
\text { Author } \\
\text { Year } \\
\text { Quality rating }\end{array}$ & $\begin{array}{l}\text { Design } \\
\text { Follow-up } \\
\text { time (FT) }\end{array}$ & $\begin{array}{l}\text { Characteristics } \\
\text { Patients, } n \\
\text { Age }\end{array}$ & $\begin{array}{l}\text { Exposure } \\
\text { Catheter type, } n \\
\text { Catheter duration } \\
\text { Catheter care }\end{array}$ & $\begin{array}{l}\text { Outcome } \\
\text { Venous thromboembolism, } n \\
\text { Diagnostic approach }\end{array}$ \\
\hline $\begin{array}{l}\text { Revel-Vilk et al } 2010 \\
\text { Fair }^{22}\end{array}$ & $\begin{array}{l}\text { Cohort, } \\
\text { multicenter } \\
\text { FT: NR }\end{array}$ & $\begin{array}{l}\text { Any cancer, } n=211 \\
\text { Age (median): } 7.4 \text { years }\end{array}$ & $\begin{array}{l}\text { Type: CVC, } n=\mathrm{NR} \text {, Port, } n=\mathrm{NR} \text {, } \\
\text { PICC, } n=\mathrm{NR} \\
\text { Duration: NR } \\
\text { Care: NR. }\end{array}$ & $\begin{array}{l}\text { Thrombosis, } n=14 \text { events in } 13 \\
\text { patients (One ALL with PICC had } \\
\text { symptomatic VTE x2) } \\
\text { Symptomatic, } n=12 \\
\text { (ALL, } n=6 \text {, non-ALL hema cancer, } \\
n=1 \text {, solid tumors, } n=5 \text { ) } \\
\text { Asymptomatic, } n=2 \\
\text { (Non-ALL hema cancer, } n=1 \text {, } \\
\text { solid tumors, } n=1 \text { ) } \\
\text { PICC related, } n=8 \\
\text { Port related, } n=1 \\
\text { CVC related, } n=1 \\
\text { Catheter removal, } n=3 \\
\text { Diagnosis: If clinically suspected, } \\
\text { confirmed by imaging. }\end{array}$ \\
\hline $\begin{array}{l}\text { Mitchell et al } 2010 \\
\operatorname{Good}^{23}\end{array}$ & $\begin{array}{l}\text { Cohort, } \\
\text { multicenter } \\
\text { FT: } 3.5 \text { months }\end{array}$ & $\begin{array}{l}\text { ALL, } n=339 \\
\text { Age (mean): } 5.9 \text { years }\end{array}$ & $\begin{array}{l}\text { Type: CVC, } n=\text { NR, Port, } n=\text { NR } \\
\text { Duration: } 3.5 \text { months } \\
\text { Care: NR } \\
\text { VTE prophylaxis: } 8 \text { of } 19 \text { patients } \\
\text { received enoxaparin before } \\
\text { catheter implantation during } \\
\text { induction therapy. }\end{array}$ & $\begin{array}{l}\text { Symptomatic, } n=19 \\
\text { Asymptomatic: NR } \\
\text { Catheter related, } n=7 \\
\text { Catheter removal: NR } \\
\text { Diagnosis: If clinically suspected, } \\
\text { confirmed by imaging. }\end{array}$ \\
\hline $\begin{array}{l}\text { Revel-Vilk et al } 2010 \\
\text { Poor }^{24}\end{array}$ & $\begin{array}{l}\text { Cohort, } \\
\text { single center } \\
\text { FT: } 0.3-7.5 \text { years }\end{array}$ & $\begin{array}{l}\text { ALL, } n=17 \\
\text { Solid tumors, } n=16 \\
\text { Non-ALL hema cancer, } n=10 \\
\text { Age: NR }\end{array}$ & $\begin{array}{l}\text { Type: } \text { CVC, } n=\mathrm{NR} \text {, Port, } n=\mathrm{NR} \text {, } \\
\text { PICC, } n=\mathrm{NR} \\
\text { Duration: NR } \\
\text { Care: NR. }\end{array}$ & $\begin{array}{l}\text { Symptomatic, } n=0 \\
\text { Asymptomatic: NR } \\
\text { Catheter related: NR } \\
\text { Catheter removal: NR } \\
\text { Diagnosis: NR. }\end{array}$ \\
\hline $\begin{array}{l}\text { Ociepa et al } 2010 \\
\text { Fair }^{25}\end{array}$ & $\begin{array}{l}\text { Cohort, } \\
\text { single center } \\
\text { FT: NR }\end{array}$ & $\begin{array}{l}\text { ALL, } n=68 \\
\text { Non-ALL hema cancer, } n=37 \\
\text { Solid tumors, } n=14 \\
\text { Any cancer, } n=5 \\
\text { Age (median): } 8.6 \text { years }\end{array}$ & $\begin{array}{l}\text { Type: CVC, } n=\mathrm{NR} \\
\text { Duration (median): } 134 \text { days } \\
\text { Care: Rinsed once weekly with } 2 \mathrm{~mL} \\
\text { of unfractionated heparin } \\
\text { ( } 50 \mathrm{U} / \mathrm{mL} \text { ) } \\
\text { VTE therapy: tPA continuously for } \\
48 \text { hours followed by LMWH in two } \\
\text { patients, and LMWH as single agent } \\
\text { in one patient. }\end{array}$ & $\begin{array}{l}\text { Symptomatic, } n=3 \\
\text { (ALL, } n=1 \text {, non-ALL hema cancer, } \\
n=1, \text { any cancer, } n=1 \text { ) } \\
\text { Asymptomatic/total, } n=4 / 37 \\
\text { Catheter related, } n=7 \\
\text { Catheter removal, } n=11 \\
\text { Diagnosis: If clinically suspected, } \\
\text { confirmed by US. In } 37 \text { patients, US } \\
\text { were performed at } 1-54 \text { months } \\
\text { after CVC removal. }\end{array}$ \\
\hline $\begin{array}{l}\text { Chung et al } 2008 \\
\text { Fair }^{26}\end{array}$ & $\begin{array}{l}\text { Cohort, } \\
\text { single center } \\
\text { FT: } 3.8 \text { years }\end{array}$ & $\begin{array}{l}\text { Solid tumors, } n=19 \\
\text { ALL, } n=11 \\
\text { Non-ALL hema cancer, } n=6 \\
\text { Age (median): } 9.1 \text { years }\end{array}$ & $\begin{array}{l}\text { Type: CVC, } n=36 \\
\text { Duration (mean): } 13 \text { months } \\
\text { Care: Flushed once weekly with } \\
\text { heparin saline and simple sterile } \\
\text { dressing was applied under aseptic } \\
\text { technique. }\end{array}$ & $\begin{array}{l}\text { Symptomatic, } n=1 \\
\text { (ALL, } n=1 \text { ) } \\
\text { Asymptomatic/total, } n=0 / 25 \\
\text { Catheter related: NR } \\
\text { Catheter removal: NR } \\
\text { Diagnosis: Evaluated clinically at } \\
\text { least once per week. If clinically } \\
\text { suspected confirmed by imaging. In } \\
25 \text { patients, venogram or CT were } \\
\text { performed at } 3-6 \text { months after } \\
\text { chemotherapy initiation. }\end{array}$ \\
\hline $\begin{array}{l}\text { Farinasso et al } 2007 \\
\text { Poor }^{27}\end{array}$ & $\begin{array}{l}\text { Cohort, } \\
\text { single center } \\
\text { FT: NR }\end{array}$ & $\begin{array}{l}\text { ALL, } n=56 \\
\text { Age (median): } 5 \text { years }\end{array}$ & $\begin{array}{l}\text { Type: CVC, } n=56 \\
\text { Duration: NR } \\
\text { Care: Flushed with } 2.5 \mathrm{~mL} \text { heparin } \\
(50 \mathrm{lU} / \mathrm{mL}) \text { three times a week. }\end{array}$ & $\begin{array}{l}\text { Symptomatic, } n=4 \text { patients/5 } \\
\text { events } \\
\text { Asymptomatic, } n=39 \\
\text { Catheter related, } n=42 \\
\text { Catheter removal: NR } \\
\text { Diagnosis: Evaluated clinically on a } \\
\text { regular basis. If clinically suspected } \\
\text { confirmed by imaging. After } \\
\text { completion of induction } \\
\text { treatment spiral CT or US was } \\
\text { performed. }\end{array}$ \\
\hline $\begin{array}{l}\text { Ruud et al } 2006 \\
\text { Fair }^{18}\end{array}$ & $\begin{array}{l}\text { RCT, } \\
\text { single blind, } \\
\text { multicenter } \\
\text { FT: } 6 \text { months }\end{array}$ & $\begin{array}{l}\text { ALL, } n=29 \text {, } \\
\text { Solid tumors, } n=22 \\
\text { Non-ALL hema cancer, } n=11 \\
\text { Age (median): } 6.7 \text { years } \\
\text { Included from same hospital } \\
\text { and same period as Ruud } \\
\text { et al. }{ }^{28} \text { Potential overlap with } \\
\text { Ruud et al. }{ }^{31}\end{array}$ & $\begin{array}{l}\text { Type: CVC, } n=48 \text {, Port, } n=14 \\
\text { Duration: } 6 \text { months } \\
\text { Care: NR } \\
\text { VTE prophylaxis: Experimental arm: } \\
\text { Warfarin (intended INR 1.3-1.9). } \\
\text { Standard arm: No prophylaxis. }\end{array}$ & $\begin{array}{l}\text { Symptomatic, } n=2 \\
\text { (non-ALL hema cancer, } n=1 \text { ) } \\
\text { Asymptomatic, } n=24 \\
\text { Catheter related: NR } \\
\text { Catheter removal: NR } \\
\text { Diagnosis: US of central neck veins } \\
\text { at 1-, 3-, and 6-month after } \\
\text { inclusion. }\end{array}$ \\
\hline
\end{tabular}


924 VTE in Pediatric Cancer Patients with CVC Hansen et al.

Table 1 (Continued)

\begin{tabular}{|c|c|c|c|c|}
\hline $\begin{array}{l}\text { Study } \\
\text { Author } \\
\text { Year } \\
\text { Quality rating }\end{array}$ & $\begin{array}{l}\text { Design } \\
\text { Follow-up } \\
\text { time (FT) }\end{array}$ & $\begin{array}{l}\text { Characteristics } \\
\text { Patients, } n \\
\text { Age }\end{array}$ & $\begin{array}{l}\text { Exposure } \\
\text { Catheter type, } n \\
\text { Catheter duration } \\
\text { Catheter care }\end{array}$ & $\begin{array}{l}\text { Outcome } \\
\text { Venous thromboembolism, } n \\
\text { Diagnostic approach }\end{array}$ \\
\hline $\begin{array}{l}\text { Ruud et al } 2006 \\
\text { Fair }^{28}\end{array}$ & $\begin{array}{l}\text { Cohort, } \\
\text { single center } \\
\text { FT: NR }\end{array}$ & $\begin{array}{l}\text { ALL, } n=30 \\
\text { Age (median): } 6 \text { years } \\
\text { Included from same hospital } \\
\text { and same period as Ruud } \\
\text { et al. }{ }^{18} \text { Potential overlap with } \\
\text { Ruud et al. }{ }^{31}\end{array}$ & $\begin{array}{l}\text { Type: CVC, } n=\mathrm{NR} \\
\text { Duration: NR } \\
\text { Care: NR. }\end{array}$ & $\begin{array}{l}\text { Symptomatic, } n=0 \\
\text { Asymptomatic, } n=15 \text { patients } \\
\text { Catheter related, } n=19 \text { events } \\
\text { Catheter-removal: NR } \\
\text { Diagnosis: US of central neck veins } \\
\text { performed just prior to asparagi- } \\
\text { nase and } 2 \text { months after asparagi- } \\
\text { nase initiation. }\end{array}$ \\
\hline $\begin{array}{l}\text { Stiakaki et al } 2005 \\
\text { Poor }^{29}\end{array}$ & $\begin{array}{l}\text { Cohort, } \\
\text { single center } \\
\text { FT: NR }\end{array}$ & $\begin{array}{l}\text { ALL, } n=19 \\
\text { Any cancer, } n=12 \\
\text { Age (median): } 4.5 \text { years }\end{array}$ & $\begin{array}{l}\text { Type: NR } \\
\text { Duration: NR } \\
\text { Care: Flushing lumen with } 2 \mathrm{~mL} \\
\text { Heplock }(10 \mathrm{IU} / \mathrm{mL}) \text { every third day } \\
\text { or after use }+ \text { weekly intraluminal } \\
\text { urokinase } 10.000 \mathrm{IU} / 2 \mathrm{~mL} \text { for } \\
4 \text { hours before aspirated or } \\
\text { discharged. }\end{array}$ & $\begin{array}{l}\text { Symptomatic, } n=1 \\
\text { (ALL, } n=1 \text { ) } \\
\text { Asymptomatic: NR } \\
\text { Catheter related, } n=1 \\
\text { Catheter removal: NR } \\
\text { Diagnosis: NR. }\end{array}$ \\
\hline $\begin{array}{l}\text { Male et al } 2003 \\
\text { Good }^{30}\end{array}$ & $\begin{array}{l}\text { Cohort, } \\
\text { multicenter } \\
\text { FT: } 4 \text { weeks }\end{array}$ & $\begin{array}{l}\text { ALL, } n=85 \\
\text { Age: NR } \\
\text { Substudy of Mitchell } 2002^{19} \\
\text { with partially overlapping } \\
\text { study population. }\end{array}$ & $\begin{array}{l}\text { Type: Catheters, } n=86, \mathrm{CVC} \text {, } \\
n=\mathrm{NR} \text {, } \\
\text { Port, } n=\mathrm{NR} \\
\text { Duration: } 4 \text { weeks } \\
\text { Care: Small amounts of unfractio- } \\
\text { nated heparin either by continuous } \\
\text { infusion }(1-3 \mathrm{IU} / \mathrm{mL}) \text { or intermit- } \\
\text { tent flushes }(50-100 \mathrm{IU} / \mathrm{mL}) \text { up to } \\
\text { four times per day. }\end{array}$ & $\begin{array}{l}\text { Symptomatic, } n=4 \\
\text { Asymptomatic, } n=25 \\
\text { Catheter related: NR } \\
\text { Catheter removal: NR } \\
\text { Diagnosis: Close clinical monitoring. } \\
\text { Confirmed by US, bilateral venog- } \\
\text { raphy, echocardiography, and MRI } \\
\text { of head. After } 4 \text { weeks, patients } \\
\text { who did not have symptoms of VTE } \\
\text { were screened with each of the four } \\
\text { radiographic tests. }\end{array}$ \\
\hline $\begin{array}{l}\text { Mitchell et al } 2003 \\
\text { Poor }^{19}\end{array}$ & $\begin{array}{l}\text { RCT } \\
\text { FT: 1-3 months }\end{array}$ & $\begin{array}{l}\text { ALL, } n=60 \\
\text { Age: } N R\end{array}$ & $\begin{array}{l}\text { Type: NR } \\
\text { Duration: } 1-3 \text { months } \\
\text { Care: NR. }\end{array}$ & $\begin{array}{l}\text { Symptomatic, } n=3 \\
\text { Asymptomatic, } n=19 \\
\text { Catheter related: NR } \\
\text { Catheter removal: NR } \\
\text { Diagnosis: Close monitoring. } \\
\text { Confirmed by radiographic test. At } \\
1-3 \text { months: echocardiography, US } \\
\text { of upper body, MRI of head. } \\
\text { Bilateral venography or MRI of the } \\
\text { upper venous system was per- } \\
\text { formed at } 1-3 \text { months. }\end{array}$ \\
\hline $\begin{array}{l}\text { Ruud et al } \\
2002 \\
\text { Good }^{31}\end{array}$ & $\begin{array}{l}\text { Cohort, } \\
\text { single center } \\
\text { FT: 3-5 months }\end{array}$ & $\begin{array}{l}\text { ALL, } n=27 \\
\text { Non-ALL hema cancer, } n=10 \\
\text { Solid tumors, } n=4 \\
\text { Age: NR } \\
\text { Included from same hospital } \\
\text { and by same author as Ruud } \\
\text { et al. }{ }^{18,28} \text { Potential overlap. }\end{array}$ & $\begin{array}{l}\text { Type: CVC, } n=41 \\
\text { Duration (mean): } 266 \text { days } \\
\text { Care: NR } \\
\text { VTE therapy: Aspiration problems } \\
\text { associated with radiology proven } \\
\text { thrombus were treated with tPA } \\
1-2 \text { mg or heparin }(1,000 \mathrm{IU} / \mathrm{mL}) \\
\text { into the CVC. }\end{array}$ & $\begin{array}{l}\text { Symptomatic, } n=0 \\
\text { Asymptomatic, } n=18 \\
\text { Catheter related: NR } \\
\text { Catheter removal, } n=0 \\
\text { Diagnosis: } 34 \text { patients had US } \\
\text { performed within days after CVC } \\
\text { insertion. At } 3-5 \text { months after first } \\
\text { examination, all patients had US } \\
\text { performed. }\end{array}$ \\
\hline $\begin{array}{l}\text { Kalmanti et al } 2002 \\
\text { Poor }^{20}\end{array}$ & $\begin{array}{l}\text { Case-control, } \\
\text { single center } \\
\text { FT: NR }\end{array}$ & $\begin{array}{l}\text { ALL, } n=14 \\
\text { Solid tumors, } n=10 \\
\text { Non-ALL hema cancer, } n=6 \\
\text { Age (mean): } 4.8 \text { years }\end{array}$ & $\begin{array}{l}\text { Type: CVC, } n=35 \\
\text { Duration (mean): } 336 \text { days } \\
\text { Care: Study: intraluminal } 2 \mathrm{~mL} \\
\text { urokinase }(5,000 \mathrm{IU} / \mathrm{mL}) \text { for } 4 \text { hours } \\
\text { once a week, in addition to } \\
\text { standard care. Control: HepLock } \\
(10 \mathrm{IU} / \mathrm{mL}) \text { flush after use or at least } \\
\text { every third day. }\end{array}$ & $\begin{array}{l}\text { Symptomatic, } n=2 \\
\text { (Non-ALL hema cancer, } n=1 \text {, } \\
\text { solid tumors, } n=1 \text { ) } \\
\text { Asymptomatic, } n=16 \\
\text { Catheter related, } n=13 \\
\text { Catheter removal: NR } \\
\text { Diagnosis: Echocardiography and } \\
\text { US regularly. If symptoms, } \\
\text { venography, or MRI angiography } \\
\text { was performed. }\end{array}$ \\
\hline $\begin{array}{l}\text { Glaser et al } 2001 \\
\text { Poor }^{32}\end{array}$ & $\begin{array}{l}\text { Cohort, } \\
\text { single center } \\
\mathrm{FT}: \geq 6 \text { months }\end{array}$ & $\begin{array}{l}\text { Solid tumors, } n=12 \\
\text { Non-ALL hema cancer, } n=7 \\
\text { ALL, } n=3 \\
\text { Age (mean): } 9.8 \text { years }\end{array}$ & $\begin{array}{l}\text { Type: Port, } n=29 \\
\text { Duration (mean): } 14 \text { months } \\
\text { Care: NR. }\end{array}$ & $\begin{array}{l}\text { Symptomatic, } n=3 \\
\text { Asymptomatic, } n=9 \\
\text { Catheter related: NR } \\
\text { Catheter removal: NR } \\
\text { Diagnosis: Physical examination and } \\
\text { contrast venography before } \\
\text { removal of catheter. }\end{array}$ \\
\hline
\end{tabular}


Table 1 (Continued)

\begin{tabular}{|c|c|c|c|c|}
\hline $\begin{array}{l}\text { Study } \\
\text { Author } \\
\text { Year } \\
\text { Quality rating }\end{array}$ & $\begin{array}{l}\text { Design } \\
\text { Follow-up } \\
\text { time (FT) }\end{array}$ & $\begin{array}{l}\text { Characteristics } \\
\text { Patients, } n \\
\text { Age }\end{array}$ & $\begin{array}{l}\text { Exposure } \\
\text { Catheter type, } n \\
\text { Catheter duration } \\
\text { Catheter care }\end{array}$ & $\begin{array}{l}\text { Outcome } \\
\text { Venous thromboembolism, } n \\
\text { Diagnostic approach }\end{array}$ \\
\hline $\begin{array}{l}\text { Rubie et al } 1995 \\
\text { Poor }^{39}\end{array}$ & $\begin{array}{l}\text { Cohort, } \\
\text { Single center } \\
\text { FT: NR }\end{array}$ & $\begin{array}{l}\text { ALL, } n=79 \\
\text { Hema cancer, } n=33 \\
\text { Solid tumors, } n=51 \\
\text { Age (median): } 85 \text { months }\end{array}$ & $\begin{array}{l}\text { CVC, } n=180 \\
\text { Duration: } 305 \text { days } \\
\text { Care: In-house: Flushed with saline } \\
\text { before infusions and with saline } \\
\text { containing } 100 \mathrm{IU} / \mathrm{mL} \text { heparin ei- } \\
\text { ther daily or after every blood } \\
\text { sampling. } \\
\text { Outpatients: flushed monthly with } \\
3-5 \mathrm{~mL} \text { diluted heparin solution } \\
(100 \mathrm{U} / \mathrm{mL}) \\
\text { VTE therapy: Urokinase } 1,000 \\
\text { IU/mL, in CVC for } 3 \text { hours. If unsuc- } \\
\text { cessful: Urokinase } 1,000 \mathrm{U} / \mathrm{mL} \text {, in } \\
\text { CVC for } 24 \text { hours }\end{array}$ & $\begin{array}{l}\text { Thrombosis/clots, } n=16 / 180 \\
\text { catheters } \\
\text { Symptomatic, } n=0 \\
\text { Asymptomatic: NR } \\
\text { Catheter related: NR } \\
\text { Catheter removal: } 4 \\
\text { Diagnosis: NR. }\end{array}$ \\
\hline $\begin{array}{l}\text { Cassey et al } 1994 \\
\text { Poor }^{4}\end{array}$ & $\begin{array}{l}\text { Cohort, } \\
\text { single center } \\
\text { FT: NR }\end{array}$ & $\begin{array}{l}\text { Any cancer, } n=10 \\
\text { Age: NR }\end{array}$ & $\begin{array}{l}\text { Type: CVC, } n=13 \\
\text { Duration (range): } 60-240 \text { days } \\
\text { Care: NR. }\end{array}$ & $\begin{array}{l}\text { Symptomatic, } n=0 \\
\text { Asymptomatic, } n=0 \\
\text { Catheter related, } n=0 \\
\text { Catheter removal, } n=0 \\
\text { Diagnosis: US at }<6 \text { months, } 6-24 \\
\text { months or }>24 \text { months. }\end{array}$ \\
\hline $\begin{array}{l}\text { Stokes et al } 1989 \\
\text { Poor }^{33}\end{array}$ & $\begin{array}{l}\text { Cohort, } \\
\text { single center } \\
\text { FT: NR }\end{array}$ & $\begin{array}{l}\text { Any cancer, } n=18 \\
\text { Age (median): } 4.9 \text { years }\end{array}$ & $\begin{array}{l}\text { Type: CVC, } n=19 \\
\text { Duration: NR } \\
\text { Care: Urokinase }(5,000 \mathrm{lU} / \mathrm{mL}) \text { into } \\
\text { the catheter for } 60 \text { minutes. } \\
\text { Afterward, flushed with } \\
\text { heparinized saline. }\end{array}$ & $\begin{array}{l}\text { Thrombosis, } n=11 \text { patients/12 } \\
\text { events } \\
\text { Symptomatic: NR } \\
\text { Asymptomatic: NR } \\
\text { Catheter related: } 12 \\
\text { Catheter removal: NR } \\
\text { Diagnosis: Catheter resistance } \\
\text { measurement. Contrast venogram } \\
\text { in } 13 \text { patients. }\end{array}$ \\
\hline $\begin{array}{l}\text { Wesenberg et al } 1987 \\
\text { Poor }^{34}\end{array}$ & $\begin{array}{l}\text { Cohort } \\
\text { FT: NR }\end{array}$ & $\begin{array}{l}\text { ALL, } n=15 \\
\text { Non-ALL hema cancer, } n=4 \\
\text { Solid tumors, } n=3 \\
\text { Age: NR }\end{array}$ & $\begin{array}{l}\text { Type: Port, } n=22 \\
\text { Duration: NR } \\
\text { Care: Flushed with } 20 \mathrm{~mL} \text { saline } \\
\text { before using and sealed with } 3 \mathrm{~mL} \\
\text { heparinized saline }(250 \mathrm{IU} / \mathrm{mL}) \\
\text { every } 4-8 \text { week. }\end{array}$ & $\begin{array}{l}\text { Symptomatic, } n=0 \\
\text { Asymptomatic, } n=0 \\
\text { Catheter related, } n=0 \\
\text { Catheter removal, } n=0 \\
\text { Diagnosis: NR. }\end{array}$ \\
\hline $\begin{array}{l}\text { Pegelow et al } 1986 \\
\text { Poor }^{35}\end{array}$ & $\begin{array}{l}\text { Cohort } \\
\text { FT: NR }\end{array}$ & $\begin{array}{l}\text { Solid tumors, } n=12 \\
\text { Hema cancer, } n=3 \\
\text { Age (mean): } 8.3 \text { years }\end{array}$ & $\begin{array}{l}\text { Type: Port, } n=16 \\
\text { Duration (mean): } 256 \text { days } \\
\text { Care: Flushed with } 5 \mathrm{~mL} \text { heparin- } \\
\text { saline after each use and at least } \\
\text { every } 3-4 \text { week. }\end{array}$ & $\begin{array}{l}\text { Thrombosis, } n=2 \\
\text { (solid tumors, } n=2 \text { ) } \\
\text { Symptomatic: NR } \\
\text { Asymptomatic: NR } \\
\text { Catheter related, } n=2 \\
\text { Catheter removal, } n=1 \\
\text { Diagnosis: NR. }\end{array}$ \\
\hline
\end{tabular}

Abbreviations: ALL, acute lymphoblastic leukemia; CT, computer tomography scan; CVC, non-tunneled or tunneled central venous catheter; Hema, hematological; INR, international normalized ratio; LMWH, low-molecular-weight heparin; MRI, magnetic resonance imaging; MRV, magnetic resonance venography; NR, not reported; PICC, peripherally inserted central catheters; Port, implantable port; RCT, randomized controlled trial; tPA, tissue plasminogen activator; US, ultrasound; VTE, venous thromboembolism; VQ, ventilation-perfusion scan.

\section{Venous Thromboembolism}

Among the 2,318 pediatric cancer patients with a catheter inserted, 400 (17\%) were diagnosed with VTE ( - Table 1). Of the 400 thrombi, 210 were asymptomatic, 142 symptomatic, and in the remaining 48 cases it was not reported whether the thrombi were asymptomatic or symptomatic. Nine studies performed systematic imaging investigations for VTE, ${ }^{18-20,28,30,31,33,40,41}$ while 13 studies closely monitored patients and if VTE was clinically suspected imaging was performed. ${ }^{17,19-23,25-27,30,32,36,37}$ Six studies performed imaging at catheter removal or study completion. ${ }^{17,25-27,32,36}$ Five studies did not report how VTE was diagnosed. ${ }^{24,29,34,35,39}$

Fifteen studies described specifically CVC-related VTE and reported 167 CVC-related VTE in 1,551 pediatric cancer patients (11\%). ${ }^{17,20,22,23,25,27-29,33-35,37,38,40,41}$ The remaining 10 studies reported VTE in children with catheters inserted but did not clearly state if the VTE was CVC related. Five studies described specifically CVC-related VTE in 459 children with ALL and reported 69 CVC-related VTE (15\%). ${ }^{23,27-29,34}$ No study described specifically CVC-related VTE in children with solid tumors.

Regarding catheter type, $20 \%$ of children with tunneled or nontunneled CVC, $20,26,27,31,33,36,37,39,41$ 12\% of children with implantable ports, ${ }^{32,34,35,37,40}$ and $4 \%$ of children with PICC $^{40}$ suffered a VTE. Twenty-one catheters were reported removed due to VTE. ${ }^{17,22,25,35,37,39}$

From 12 studies reporting VTE in ALL children, the overall incidence $(95 \% \mathrm{CI})$ for VTE, symptomatic or asymptomatic, was $21 \%$ (8-37) (- Fig. 2A). ${ }^{19,21,23-30,34,38}$

Nine studies reported VTE in children with solid tumors with an overall incidence $(95 \% \mathrm{CI})$ for symptomatic or asymptomatic VTE at 7\% (0.1-17.0) (- Fig. 2B). ${ }^{17,21,24,26,34,35,37,38,40}$ Funnel plot 

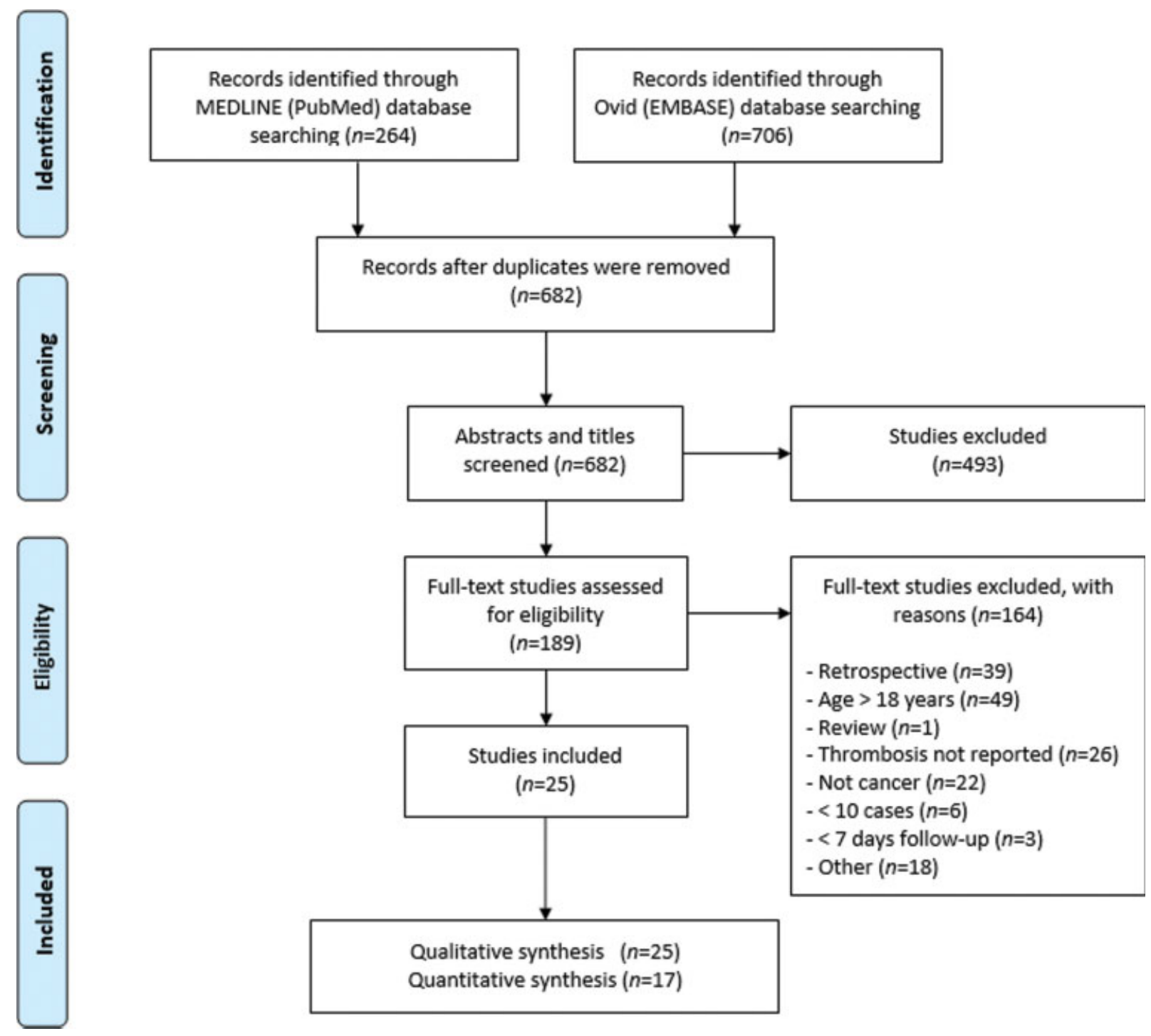

Fig. 1 PRISMA flow diagram of the inclusion and exclusion process of articles. ${ }^{11}$

was asymmetric for both children with ALL and solid tumors studies ( Fig. 3A, B) indicating low publication bias. The $I^{2}$ for ALL studies was $95 \%$ and for solid tumors studies it was $88 \%$, indicating considerable heterogeneity between included studies. ${ }^{14}$

\section{Catheter Care and Thromboprophylaxis}

Half of the included studies described how the indwelled catheters were cared for. ${ }^{17,20,25-27,29,30,33-35,37,39}$

Catheter care consisted of heparin lock $17,25,34$ or flushes $^{20,26,27,30,34,35,37,39}$ in 10 studies (976 children), ${ }^{17,20,25-27,30,34,35,37,39}$ urokinase instillation in one study ( 18 children), ${ }^{33}$ and heparin flushes plus urokinase instillation in two studies (46 children). ${ }^{20,29}$ Despite catheter care, VTE was diagnosed in 130 (13\%) children with heparin, 11 (61\%) patients with urokinase, and eight (17\%) children with heparin flushes plus urokinase instillation as localized catheter care.

Schoot et al ${ }^{17}$ compared $70 \%$ ethanol with $100 \mathrm{IU} / \mathrm{mL}$ heparin as lock solution and found no statistically significant difference (three symptomatic VTE in 152 children with ethanol as lock solution vs. no symptomatic VTE in 153 children with heparin as lock solution $(p=0.25))$. In addition,
$2 \%$ of children with ethanol as lock solution versus $5 \%$ of children with heparin as lock solution were diagnosed with asymptomatic VTE $(p=0.17)$. Additionally, Kalmanti et al ${ }^{20}$ compared intraluminal urokinase in addition to heparin (study) versus standard heparin care (control) and found asymptomatic VTE in seven of 19 study children and nine of 11 control children $(p=0.047)$.

Three studies investigated thromboprophylaxis in addition to lock solution. ${ }^{18,23,37}$ Ruud et al ${ }^{18}$ compared warfarin (intended INR range 1.3-1.9) with no prophylaxis and found no difference in number of VTE $(p=0.44)$. Mitchell et $\mathrm{al}^{23}$ compared enoxaparin $(1 \mathrm{mg} / \mathrm{kg})$ before catheter insertion $(n=8)$ to no prophylaxis before catheter insertion $(n=11)$ in high-risk ALL patients and found that one child receiving enoxaparin $1 \mathrm{mg} / \mathrm{kg}$ experienced a VTE compared with eight VTE cases out of 11 children without prophylaxis $(p=0.023)$. Abate et $\mathrm{al}^{37}$ provided low-molecular weight heparin (LMWH) to 85 of 155 included children and reported that one out of the 85 children receiving LMWH suffered symptomatic VTE. However, asymptomatic VTE was not reported by Abate et al. ${ }^{37}$ 

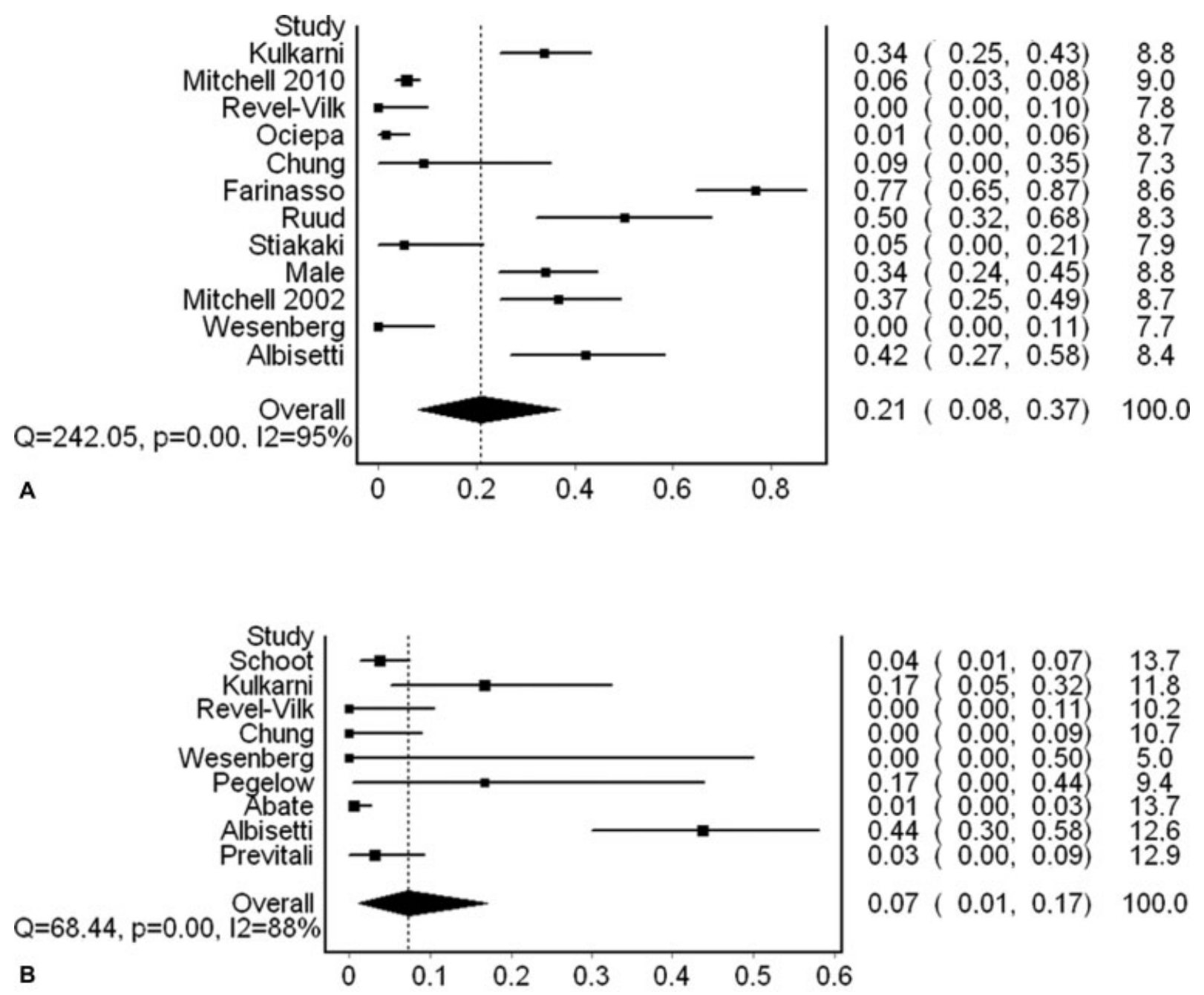

Fig. 2 Forest plots of incidence of symptomatic or asymptomatic venous thromboembolism in children suffering from acute lymphoblastic leukemia, based on 12 studies (A), or solid tumors, based on nine studies (B).

\section{Thrombophilia}

Eight studies investigated thrombophilia (- Supplementary Table S1, available in the online version only). ${ }^{18,19,22,26,29-31,38}$ Six of 11 (55\%) children with persistent antiphospholipid antibodies, ${ }^{19,30}$ three of $15(20 \%)$ children with the factor $\mathrm{V}$ Leiden variation, ${ }^{19,29,30,38}$ and one of 25 (4\%) children with the methylenetetrahydrofolate reductase variation $^{26}$ suffered a VTE. None of the children with the factor II variation $(n=7),{ }^{19,26,29,30,38}$ protein C deficiency $(n=2),{ }^{26}$ or protein S deficiency $(n=1)^{26}$ suffered a VTE. Three of 75 ( $\left.4 \%\right)$ children without thrombophilia suffered a VTE. ${ }^{18,26,29}$ However, Revel-Vilk et al only investigated thrombophilia in children suffering VTE $(n=13)$, and found that eight $(62 \%)$ children with VTE did not have thrombophilia. ${ }^{22}$ None of the eight studies reported a significant association between the presence of thrombophilia and VTE.

\section{Discussion}

To the best of our knowledge, this is the first systematic review to disclose the literature on VTE in pediatric cancer patients with CVC inserted. From 25 studies including a total of 2,318 pediatric cancer patients with a catheter inserted, we found that 400 (17\%) were diagnosed with VTE. From 15 studies specifically reporting CVC-related VTE an incidence of $11 \%$ was found. The overall estimated VTE incidence was substantially higher in children with ALL (21\%) than children with solid tumors (7\%). Additionally, children with tunneled or nontunneled CVC were more likely to suffer from VTE than children with implantable ports.

Of the 25 included studies, 13 had acceptable quality (rating good or fair). Of the 12 studies with poor quality, the majority lacked information on sample size, power calculation, outcome assessment, or study population. Altogether, a good or fair quality in $52 \%$ of included studies is acceptable, but it should be taken into account when interpreting the results and conclusion of this review

To reach a compromise between sufficient sample size and reduction of heterogeneity, we divided the study population into ALL, non-ALL hematological cancer, solid tumors, any cancer and any hematological cancer if the number of ALL cases was not specified. The solid tumor group constituted a wide range of cancer diagnosis, which limits its usefulness for specific diagnosis and increases its heterogeneity but increases the generalizability and made a meta-analysis possible. 

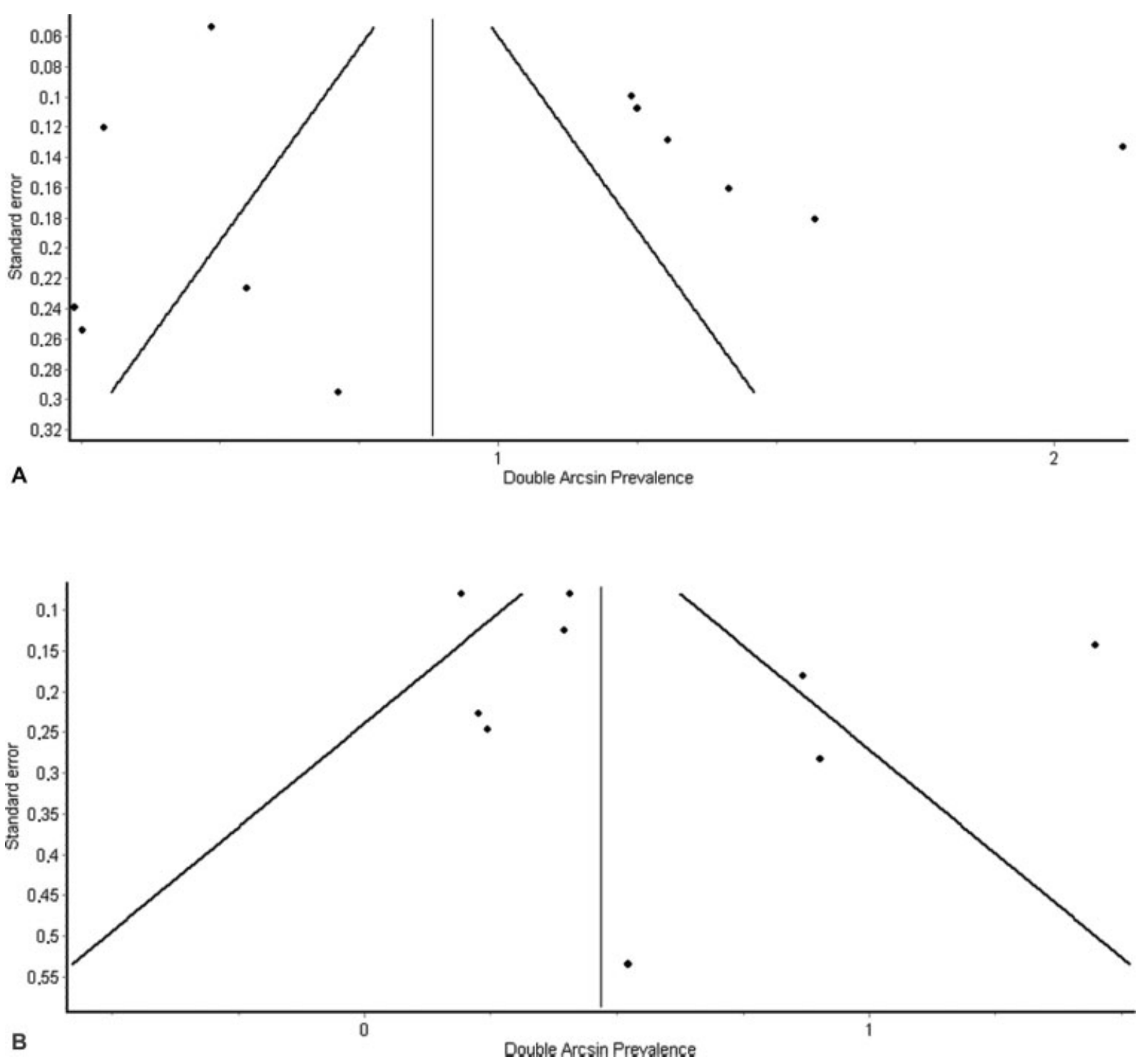

Fig. 3 Funnel plot of incidence of symptomatic or asymptomatic venous thromboembolism in children suffering from acute lymphoblastic leukemia, based on 12 studies (A), or solid tumors, based on nine studies (B).

The incidence of CVC-related VTE in adults with cancer has been reported to range from 0.3 to $28.3 \%{ }^{42}$ We found that $11 \%$ of pediatric cancer patients with a catheter inserted suffered from CVC-related VTE, which is considered equal to that of adults. ${ }^{9,10}$ The incidence of CVC-related VTE in adults with ALL has been reported to range from 6.5 to $11.7 \%,{ }^{43,44}$ and from 3.8 to $13 \%$ in adults with solid tumors and a catheter. ${ }^{45,46}$ Five studies described specifically CVC-related VTE in 459 children with ALL and 69 CVC-related VTE (15\%). ${ }^{23,27-29,34}$ Unfortunately, no study described specifically CVC-related VTE in children with solid tumors. The statistical dispersion of our estimated VTE incidence was wide for both ALL and solid tumors, and therefore the VTE estimates should be interpreted with caution. However, the present meta-analyses suggest that children with ALL and a catheter have a higher incidence of VTE than adults with ALL and a catheter.

The VTE incidence in children with ALL and a CVC inserted (21\%) is considerably higher than the overall thrombosis rate of $5.2 \%$ found in a previous meta-analysis of 1,752 children with
ALL. ${ }^{47}$ However, only few of the studies included by Caruso et al ${ }^{47}$ describe CVC, but $27.5 \%$ of the reported symptomatic thrombi were catheter related, which is higher than our finding of $15 \%$. Nevertheless, this supports the fact that CVC indeed is a severe additional thromboembolic risk factor in children with ALL.

Regarding catheter type, the majority of patients had either tunneled CVC, nontunneled CVC or implantable ports inserted, whereas only $2.4 \%$ of children had PICC. Revel-Vilk et $\mathrm{al}^{22}$ found eight PICC-related VTE in 211 patients, but the number of PICC in the 211 patients was not reported. Thus, the VTE risk of PICC cannot be concluded from that study.

Catheter care was only reported explicitly in half of the included studies. ${ }^{17,20,25-27,29,30,33-35,37,39}$ Among these, 976 of 1,040 children received heparin as catheter care. Although we found a VTE incidence of $61 \%$ in children with urokinase as catheter care, and only $13 \%$ in children with heparin as catheter care, we render that studies with direct comparison of different catheter care regimens are warranted to conclude which regimen is superior to the others. 
Testing for inherited thrombophilia in children is in general debatable. ${ }^{48}$ However, several studies have found that inherited thrombophilia does not increase the risk of CVC-related VTE in children. ${ }^{48}$ Accordingly, none of the eight studies we identified investigating thrombophilia in pediatric cancer patients reported a significant association between the presence of thrombophilia and VTE. ${ }^{18,19,22,26,29-31,38}$ Altogether, thrombophilia testing does not seem recommendable in pediatric cancer patients with CVC and VTE.

The strengths of the present systematic review are the comprehensive literature search and the inclusion of a high number of pediatric cancer patients. Consequently, the number of VTE was sufficient for statistical purposes. We only included prospective studies, which ensured systematic data collection. Studies included in the meta-analysis were evenly distributed in the funnel plot, especially the one for ALL indicating a low publication bias of the included studies.

However, some limitations must be considered. The included studies were quite heterogeneous, indicated by $I^{2}$ at $95 \%$ for ALL studies and $88 \%$ for solid tumor studies. Not all studies reported asymptomatic VTE, which is much more incident than symptomatic VTE, 27 and therefore the VTE incidence could be underestimated. This is due to the design of the included studies, where only nine studies performed systematic imaging investigations for VTE. We reported VTE per patient, since the total number of included catheters could not be calculated. As some patients may need catheter replacement, VTE per catheter would have been useful information. Rubie et $\mathrm{al}^{39}$ did only report VTE per catheter and found that 16 of 180 (9\%) tunneled or nontunneled CVC catheters had VTE. Partially overlapping study populations existed between Male et $\mathrm{al}^{30}$ and Mitchell et al. ${ }^{19}$ Moreover, there was potential overlap of three study populations included from the same hospital and by the same author, ${ }^{18,28,31}$ with two of them also including patients from the same time period. ${ }^{18,28}$ In case of overlapping study populations, children are counted twice, and consequently drive the results in the same direction and thereby introduce bias.

\section{Conclusion}

Children with ALL and CVC hold a higher VTE incidence compared with children with solid tumors and CVC. Importantly, pediatric cancer patients with implantable port catheters probably have lower risk of VTE than children with tunneled or nontunneled CVC. Investigation for thrombophilia does not seem relevant in pediatric cancer patients with CVC and VTE. Intensified catheter care is recommended, especially in children with ALL. Finally, implantable port catheter should be preferred to reduce the VTE risk.

Funding

None.

\section{Conflict of Interest}

A.-M.H. reports other from CSL Behring, other from CSL Behring, Bayer, Boehringer Ingelheim, Astellas, outside the submitted work.

\section{References}

1 Steliarova-Foucher E, Colombet M, Ries LAG, et al; IICC-3 contributors. International incidence of childhood cancer, 2001-10: a populationbased registry study. Lancet Oncol 2017;18(06):719-731

2 Trousseau A. Clinique Medicale de l'Hotel-Dieu Paris. London: The New Sydenham Society. Phlegmasia alba dolens; 1865: 654-712

3 Forbrigger Z, Kuhle S, Brown MM, Moorehead PC, Digout C, Kulkarni K. The association of venous thromboembolism with survival in pediatric cancer patients: a population-based cohort study. Ann Hematol 2018;97(10):1903-1908

4 Athale U, Siciliano S, Thabane L, et al. Epidemiology and clinical risk factors predisposing to thromboembolism in children with cancer. Pediatr Blood Cancer 2008;51(06):792-797

5 Ko RH, Thornburg CD. Venous Thromboembolism in Children with Cancer and Blood Disorders. Front Pediatr 2017;5:12

6 Bordbar M, Karimi M, Shakibazad N. Thrombosis in pediatric malignancy: a review and future perspectives with focus on management. Blood Coagul Fibrinolysis 2018;29(07):596-601

7 Onyeama SN, Hanson SJ, Dasgupta M, Baker K, Simpson PM, Punzalan RC. Central venous catheter-associated venous thromboembolism in children with hematologic malignancy. J Pediatr Hematol Oncol 2018;40(08):e519-e524

8 Giordano P, Saracco P, Grassi M, et al; Italian Association of Pediatric Hematology and Oncology (AIEOP) Recommendations for the use of long-term central venous catheter (CVC) in children with hemato-oncological disorders: management of CVC-related occlusion and CVC-related thrombosis. On behalf of the coagulation defects working group and the supportive therapy working group of the Italian Association of Pediatric Hematology and Oncology (AIEOP). Ann Hematol 2015;94(11):1765-1776

9 Chopra V, Anand S, Hickner A, et al. Risk of venous thromboembolism associated with peripherally inserted central catheters: a systematic review and meta-analysis. Lancet 2013;382 (9889):311-325

10 Ahn DH, Illum HB, Wang DH, Sharma A, Dowell JE. Upper extremity venous thrombosis in patients with cancer with peripherally inserted central venous catheters: a retrospective analysis of risk factors. J Oncol Pract 2013;9(01):e8-e12

11 Moher D, Liberati A, Tetzlaff J, Altman DGPRISMA Group. Preferred reporting items for systematic reviews and meta-analyses: the PRISMA statement. Ann Intern Med 2009;151(04):264-269, W64

12 National Heart, Lung, and Blood Institute. Study Quality Assessment Tools . Accessed February 19, 2021 at: https://www.nhlbi. nih.gov/health-topics/study-quality-assessment-tools

13 Barendregt JJ, Doi SA, Lee YY, Norman RE, Vos T. Meta-analysis of prevalence. J Epidemiol Community Health 2013;67(11): 974-978

14 Higgins JPT, Thompson SG, Deeks JJ, Altman DG. Measuring inconsistency in meta-analyses. BMJ 2003;327(7414):557-560

15 Higgins JP, Thompson SG. Quantifying heterogeneity in a metaanalysis. Stat Med 2002;21(11):1539-1558

16 Terrin N, Schmid CH, Lau J, Olkin I. Adjusting for publication bias in the presence of heterogeneity. Stat Med 2003;22(13):2113-2126

17 Schoot RA, van de Wetering MD, Stijnen T, et al; DCOG-Aristocaths Supportive Care Working Group. Prevalence of symptomatic and asymptomatic thrombosis in pediatric oncology patients with tunneled central venous catheters. Pediatr Blood Cancer 2016;63 (08):1438-1444

18 Ruud E, Holmstrøm H, De Lange C, Hogstad EM, Wesenberg F. Low-dose warfarin for the prevention of central line-associated thromboses in children with malignancies-a randomized, controlled study. Acta Paediatr 2006;95(09):1053-1059

19 Mitchell LG, Andrew M, Hanna K, et al; Prophylactic Antithrombin Replacement in Kids with Acute Lymphoblastic Leukemia Treated with Asparaginase Group (PARKAA) A prospective cohort study determining the prevalence of thrombotic events in children with 
acute lymphoblastic leukemia and a central venous line who are treated with L-asparaginase: results of the Prophylactic Antithrombin Replacement in Kids with Acute Lymphoblastic Leukemia Treated with Asparaginase (PARKAA) Study. Cancer 2003;97(02):508-516

20 Kalmanti M, Germanakis J, Stiakaki E, et al. Prophylaxis with urokinase in pediatric oncology patients with central venous catheters. Pediatr Hematol Oncol 2002;19(03):173-179

21 Kulkarni K, Halton J, Spavor M, et al. Increased requirement for central venous catheter replacement in paediatric oncology patients with deep venous thrombosis: a multicentre study. Thromb Haemost 2015;113(02):434-435

22 Revel-Vilk S, Yacobovich J, Tamary H, et al. Risk factors for central venous catheter thrombotic complications in children and adolescents with cancer. Cancer 2010;116(17):4197-4205

23 Mitchell L, Lambers M, Flege S, et al. Validation of a predictive model for identifying an increased risk for thromboembolism in children with acute lymphoblastic leukemia: results of a multicenter cohort study. Blood 2010;115(24):4999-5004

24 Revel-Vilk S, Menahem M, Stoffer C, Weintraub M. Post-thrombotic syndrome after central venous catheter removal in childhood cancer survivors is associated with a history of obstruction. Pediatr Blood Cancer 2010;55(01):153-156

25 Ociepa T, Maloney E, Urasinski T, Sawicki M. Thrombotic complications of tunneled central lines in children with malignancy. J Pediatr Hematol Oncol 2010;32(02):88-92

26 Chung BH, Ma ES, Khong PL, Chan GC. Inherited thrombophilic factors do not increase central venous catheter blockage in children with malignancy. Pediatr Blood Cancer 2008;51(04):509-512

27 Farinasso L, Bertorello N, Garbarini L, et al. Risk factors of central venous lines-related thrombosis in children with acute lymphoblastic leukemia during induction therapy: a prospective study. Leukemia 2007;21(03):552-556

28 Ruud E, Holmstrøm H, de Lange C, Natvig S, Albertsen BK, Wesenberg F. Thrombotic effects of asparaginase in two acute lymphoblastic leukemia protocols (NOPHO ALL-1992 versus NOPHO ALL-2000): a single-institution study. Pediatr Hematol Oncol 2006;23(03):207-216

29 Stiakaki E, Germanakis I, Sfyridaki C, Katzilakis N, Danilatou V, Kalmanti M. Prevalence of Factor V Leiden and other thrombophilic traits among Cretan children with malignancy. Pediatr Blood Cancer 2005;44(04):386-389

30 Male C, Chait P, Andrew M, Hanna K, Julian J, Mitchell LPARKAA Investigators. Central venous line-related thrombosis in children: association with central venous line location and insertion technique. Blood 2003;101(11):4273-4278

31 Ruud E, Holmstrøm H, Natvig S, Wesenberg F. Prevalence of thrombophilia and central venous catheter-associated neck vein thrombosis in 41 children with cancer-a prospective study. Med Pediatr Oncol 2002;38(06):405-410

32 Glaser DW, Medeiros D, Rollins N, Buchanan GR. Catheter-related thrombosis in children with cancer. J Pediatr 2001;138(02):255-259

33 Stokes DC, Rao BN, Mirro J Jr, et al. Early detection and simplified management of obstructed Hickman and Broviac catheters. J Pediatr Surg 1989;24(03):257-262
34 Wesenberg F, Anker C, Sommerschild H, Flaatten H. Central venous catheter with subcutaneous injection port (Port-ACath): clinical experience with children. Pediatr Hematol Oncol 1987;4(02):137-143

35 Pegelow CH, Narvaez M, Toledano SR, Davis J, Oiticica C, Buckner D. Experience with a totally implantable venous device in children. Am J Dis Child 1986;140(01):69-71

36 Östlund Å, Fläring U, Norberg Å, et al. Incidence of and risk factors for venous thrombosis in children with percutaneous non-tunnelled central venous catheters. Br J Anaesth 2019;123(03):316-324

37 Abate ME, Sánchez OE, Boschi R, et al. Analysis of risk factors for central venous catheter-related complications: a prospective observational study in pediatric patients with bone sarcomas. Cancer Nurs 2014;37(04):292-298

38 Albisetti M, Kellenberger CJ, Bergsträsser E, et al. Port-a-cathrelated thrombosis and postthrombotic syndrome in pediatric oncology patients. J Pediatr 2013;163(05):1340-1346

39 Rubie H, Juricic M, Claeyssens S, et al. Morbidity using subcutaneous ports and efficacy of vancomycin flushing in cancer. Arch Dis Child 1995;72(04):325-329

40 Previtali P, Paladini S, Gandini L, et al. Role of serial ultrasound screening of venous thrombosis in oncologic children with central lines. Pediatr Hematol Oncol J 2019;4:1-6

41 Cassey J, Hendry GM, Patel J. Evaluation of long-term central venous patency in children with chronic venous catheters using image-directed Doppler ultrasonography. J Clin Ultrasound 1994; 22(05):313-315

42 Kahale LA, Tsolakian IG, Hakoum MB, et al. Anticoagulation for people with cancer and central venous catheters. Cochrane Database Syst Rev 2018;6(06):CD006468

43 Htun KT, Ma MJY, Lee AYY. Incidence and outcomes of catheter related thrombosis (CRT) in patients with acute leukemia using a plateletadjusted low molecular weight heparin regimen. J Thromb Thrombolysis 2018;46(03):386-392

44 Refaei M, Fernandes B, Brandwein J, Goodyear MD, Pokhrel A, Wu C. Incidence of catheter-related thrombosis in acute leukemia patients: a comparative, retrospective study of the safety of peripherally inserted vs. centrally inserted central venous catheters. Ann Hematol 2016;95(12):2057-2064

45 Al-Asadi O, Almusarhed M, Eldeeb H. Predictive risk factors of venous thromboembolism (VTE) associated with peripherally inserted central catheters (PICC) in ambulant solid cancer patients: retrospective single Centre cohort study. Thromb J 2019;17:2

46 Decousus H, Bourmaud A, Fournel P, et al; ONCOCIP Investigators. Cancer-associated thrombosis in patients with implanted ports: a prospective multicenter French cohort study (ONCOCIP). Blood 2018;132(07):707-716

47 Caruso V, Iacoviello L, Di Castelnuovo A, et al. Thrombotic complications in childhood acute lymphoblastic leukemia: a metaanalysis of 17 prospective studies comprising 1752 pediatric patients. Blood 2006;108(07):2216-2222

48 Raffini L. Thrombophilia in children: who to test, how, when, and why? Hematology (Am Soc Hematol Educ Program) 2008; 2008:228-235 